\title{
The influence of cooperation and defection on social decision making in depression: A study of the iterated prisoner's dilemma game.
}

\author{
Kristen M. Sorgi, Mascha van 't Wout * \\ Department of Cognitive, Linguistic and Psychological Sciences, Brown University \\ 190 Thayer Street, Providence, RI, 02912, United States \\ Department of Psychiatry and Human Behavior, Alpert Medical School of Brown University \\ 345 Blackstone Boulevard, Providence, RI, 02906, United States
}

\footnotetext{
* Corresponding Author: Mascha van 't Wout. Department of Psychiatry and Human Behavior. Alpert Medical School of Brown University. 345 Blackstone Blvd. Providence, RI 02906, USA. Email: maschavantwout@gmail.com |Phone: 401-863-6891 |Fax: 401-455-6441
} 


\section{Abstract}

This study evaluated the influence of self-reported levels of depression on interpersonal strategic decision making when interacting with partners who differed in their predetermined tendency to cooperate in three separate computerized iterated Prisoner's Dilemma Games (iPDGs). Across 29 participants, cooperation was lowest when interacting with a predominantly defecting partner and highest when interacting with a predominantly cooperating partner. Greater depression severity was related to steadier and continued cooperation over trials with the cooperating partner, seeming to reflect a prosocial response tendency when interacting with this partner. With the unbiased partner, depression severity was associated with a more volatile response pattern in reaction to cooperation and defection by this partner. Severity of depression did not influence cooperation with a defecting partner or expectations about partner cooperation reported before the task began. Taken together, these data appear to show that in predominately positive interactions, as in the cooperating partner condition, depression is associated with less volatile, more consistent cooperation. When such clear feedback is absent, as in the unbiased partner condition, depression is associated with more volatile behavior. Nonetheless, participants were generally able to adapt their behavior accordingly in this dynamic interpersonal decision making context.

Keywords: Interpersonal; bias; learning; emotion; negative affect; anxiety 


\section{Introduction}

Depression is a prevalent mental disorder characterized by dysphoric mood, loss of interest or pleasure in activities, and accompanying symptoms such as hopelessness and low energy, which together cause significant distress or impairment in normal functioning (American Psychiatric Association, 2013). Depression has been associated with various affective and cognitive distortions and underlying neurobiological changes (Clark et al., 2009). For instance, clinically depressed individuals have shown impairments in recognizing happy facial expressions (Rubinow and Post, 1992), as well as faster responding to sad versus happy targets in an affective go/no-go task (Erickson et al. 2005; Murphy et al., 1999). Depression has further been associated with a memory bias favoring the recall of negative information (Brittlebank et al., 1993; Joormann et al., 2009), as well as the tendency to underestimate instances of reward and to overestimate both instances of punishment (Nelson and Craighead, 1977) and the likelihood of failing after negative performance feedback (Elliott et al., 1997). This orientation toward negatively valenced stimuli and behavioral feedback is likely to have implications for how depressed individuals respond to emotionally charged information in social situations (Joiner, 1995; Nepon et al., 2011).

Theorists studying interpersonal behavior have focused on the importance of an individual's efficacy as a group member (Argyris, 1965) and the ability to adapt behaviors to obtain desirable social outcomes (Libet and Lewinsohn, 1973). This highlights the importance of strategic decision making in interpersonal contexts, which can be quantified by paradigms borrowed from behavioral game theory. In a task known as the iterated Prisoner's Dilemma Game (iPDG; Axelrod and Hamilton, 1981), each of two players works toward the supposed goal of earning points or money by choosing independently to cooperate or defect over the course of multiple trials. Defecting while one's partner cooperates results in the largest individual payoff for the defector but a zero payoff for the cooperator. Mutual cooperation results in second-highest, but equal, individual payoffs. Mutual defection results in equal, but second- 
lowest, individual payoffs. Given this scenario, choosing to cooperate is risky, because the other player may choose to defect and in doing so will gain the highest individual pay-off, leaving you with nothing. Choosing to defect, on the other hand, results in a sure pay-off, the value of which will be higher or lower depending on whether the other player decides to cooperate or defect, respectively. For this reason, the optimal game theoretical decision would be for both players to defect, even though this amounts to a suboptimal outcome because the earnings for both players would be higher if both decided to cooperate. Despite the risk associated with choosing to cooperate, cooperative play frequently occurs, even in situations where individuals interact only once (Berg et al., 1995; Camerer, 2003; Sally, 1995), and allows each player to establish a positive reputation, resulting in the more advantageous option of reciprocal cooperation over multiple iterations of the game (Andreoni and Miller, 1993). Thus, whether an individual is willing to make a risky social decision (e.g. to cooperate) or not (e.g. to defect) is influenced by how the other player has acted previously (Nowak, 2006). Therefore, players' decisions are influenced by how they interpret, respond to, and learn from this type of social information.

Alterations in social information processing due to cognitive distortions, such as those associated with depression, may result in aberrant strategic decision making in social contexts (Harlé et al., 2010). For instance, McClure et al. (2007) found that adolescents with anxiety and depressive disorders responded more cooperatively in an IPDG to cooperative partners compared to adolescents without these disorders. In contrast, Surbey (2011) found a negative correlation between intentions to cooperate in a vignette-based PDG task and severity of participants' self-reported depression symptoms. Similarly, Clark et al. (2013) reported an association between low levels of self-reported depression and a reduced tendency to maintain mutual cooperation in an iPDG, and Pulcu et al. (2015) reported that participants with current clinical depression defected significantly more in an IPDG than either healthy controls or those with depression in remission. Taken together, previous research investigating depression and PDG play appears to favor an association between depression and non-cooperative behavior. 
However, an important aspect of social behavior is the repeated nature of interactions, and expectations about another person's behavior guide future interactions. More specifically, having positive or negative social and moral perceptions of another person influences a player's willingness to make a risky decision to cooperate and trust that person (Delgado et al., 2005; van 't Wout and Sanfey, 2008). Furthermore, Chang et al. (2010) demonstrated that although this willingness to make a cooperative decision to trust someone may initially depend on implicit beliefs about reciprocation, these beliefs can be updated based on repeated interactions. However, to the best of our knowledge, no previous studies have assessed the relationship between depression and the ability to adapt to varying cooperative response patterns over the course of repeated interactions in an iPDG. We thus aimed to extend previous research by implementing an IPDG in which participants may adapt their cooperation based on experience gained when repeatedly interacting with different partners. In particular, the present study evaluated repeated decision making in individuals reporting different levels of depression severity when confronted with three partners who differed in their predetermined tendency to cooperate (or defect) in separate iPDGs. Based on prior research associating depression with biases toward negative information in learning and memory (e.g. Joormann et al., 2009; Nelson and Craighead, 1977), in addition to the literature discussed above linking depression to higher average defection in the PDG, we hypothesized that depressive symptoms would be associated with a more rapid transition to defecting as a response tendency when interacting with a partner who mostly defected, which is a negatively valenced social interaction.

We did not anticipate depression to impact decisions to cooperate when repeatedly interacting with a partner who mostly cooperated due to the strategic options available when playing the iPDG with such a partner. Namely, we expected that participants would equally recognize the benefit of mutual cooperation--resulting in the decision to cooperate--and the potential to personally gain by defecting against such a partner--resulting in the decision to defect. We further evaluated whether there was a relationship between levels of depression and 
expectations about partner cooperation before participants played the games. Given the typically high comorbidity between depression and anxiety symptoms (Kessler et al., 2005), we also assessed the influence of self-reported anxiety symptoms on decision making in these iPDGs.

We further hypothesized that participants, regardless of self-reported depression levels, would alter their response patterns to reflect the general response tendency of their partners over repeated trials. For instance, participants may follow so called 'tit-for-tat-like' or 'win-stay, lose-shift-like' strategies (Nowak and Sigmund, 1993). Thus, we expected that participants would transition to the lowest level of cooperation when interacting with a partner who mostly defected, acquire the highest cooperation when interacting with a partner who mostly cooperated, and maintain intermediate cooperation when interacting with a partner who did not display a clear preference for cooperation or defection (i.e. cooperating $50 \%$ of time).

Following an approach similar to those of prior studies (Clark et al., 2013; Haley and Strickland, 1986; Surbey, 2011), we assessed self-reported severity of depression to reflect the idea that depression is a continuum rather than an all-or-nothing phenomenon. Many people will experience symptoms of depression to varying degrees throughout their lives without actually meeting clinical criteria for a depressive disorder. Although symptoms may not meet the severity of clinical threshold, behavioral changes may be noted (Judd et al., 1996). Investigating a population including individuals meeting criteria for depression as well as those with subclinical symptom levels can provide important insight into behavioral alterations through a more inclusive lens.

\section{Methods}

\subsection{Participants}

Thirty-one participants were recruited from the Providence, Rhode Island metro area by online advertisements. Exclusion criteria were current alcohol or drug dependence, history of a 
psychotic episode or disorder, neurological conditions (e.g. head trauma, loss of consciousness), and age above 60 years. To ensure recruitment of individuals along the range of depression/anxiety symptoms, we recruited participants with and without a history of depression/anxiety.

After signing informed consent, all participants completed the Mini International Neuropsychiatric Interview (MINI) 5.0.0 for DSM-IV (Sheehan et al., 1998) to determine the presence of depression and/or other mental health disorders. Two participants were excluded from the sample due to responding affirmatively to exclusion criteria (including, but not limited to, substance dependence and psychotic disorders), resulting in a group of 29 participants. This experiment was part of a larger research study on understanding the connections between depression and economic behaviors funded by NSF, and participants received monetary compensation based on their performance on components of the entire experimental procedure in addition to a show-up fee. The Brown University Institutional Review Board (IRB) approved all study materials and procedures, consistent with the Declaration of Helsinki.

\subsection{Materials}

\subsubsection{Self-reported depression and anxiety}

Self-reported depression levels were assessed with the Beck Depression Inventory (BDI; Beck et al., 1979). One question on suicidality, item 9, was removed due to IRB regulations. Given its high comorbidity with depression, self-reported levels of anxiety were assessed using the Beck Anxiety Inventory (BAI; Beck and Steer, 1990).

\subsubsection{Estimate of intellectual functioning}

The Vocabulary and Matrix Reasoning subtests of the Wechsler Abbreviated Scale of Intelligence (WASI; Psychological Corporation, 1999) were administered in order to estimate 
crystallized and fluid intelligence, respectively. The combination of these two test scores is considered a valid indicator of general intelligence (Canivez et al., 2009).

\subsubsection{Iterated Prisoner's Dilemma Game}

Participants completed three versions of an adapted computerized iterated Prisoner's Dilemma Game (iPDG; Axelrod and Hamilton, 1981) programmed using E-Prime 2.0 software

(Psychology Software Tools, Pittsburgh, PA). In one version, participants played the iPDG with a partner who cooperated on $50 \%$ and defected on $50 \%$ of trials, i.e. unbiased partner. In a second version, participants interacted with a partner who cooperated on $25 \%$ and defected on $75 \%$ of trials, i.e. defecting partner. In a third version, participants played with a partner who cooperated on $75 \%$ and defected on $25 \%$ of trials, i.e. cooperating partner. Participants were told that they would play multiple trials of the iPDG with each of three partners (i.e. three separate games, each with a single partner), and that each partner would have no knowledge of the responses made during the trials played with the other partners.

In each of the three iPDGs, participants played with a single partner for 12 trials, which previous research has demonstrated is sufficient for individuals to achieve stable rates of cooperation (Chang et al., 2010). The order of trials on which the partner choose to cooperate (C) or defect (D) in each iPDG version was fixed. The order of partner cooperation/defection trials for the unbiased partner was: $C ; D ; C ; C ; D ; D ; C ; D ; C ; D ; C ; D$. For the cooperating partner, the order was: $\mathrm{C} ; \mathrm{C} ; \mathrm{C} ; \mathrm{D} ; \mathrm{C} ; \mathrm{C} ; \mathrm{C} ; \mathrm{D} ; \mathrm{C} ; \mathrm{C} ; \mathrm{C} ; \mathrm{D}$. For the defecting partner, the order was: $\mathrm{C} ; \mathrm{D} ; \mathrm{D} ; \mathrm{C} ; \mathrm{D} ; \mathrm{D} ; \mathrm{D} ; \mathrm{D} ; \mathrm{C} ; \mathrm{D} ; \mathrm{D} ; \mathrm{D}$. All participants played all three iPDG versions, which were presented in a pseudorandomized order among participants to control for order effects. Since previous research indicates that, despite gender effects on decisions in the IPDG, cooperation rates are comparable in single-gender games (Ortmann and Tichy, 1999), two identical versions of each iPDG were created such that participants only played with partners of their own gender. 
At the start of the task, the experimenter provided instructions, and participants were shown a sample payoff matrix and how to respond. See Supplementary Materials for a description of these instructions and a screenshot of one iPDG trial. Participants were shown a photograph and the name of their partner at the start of each trial. Next, they saw the payoff matrix and a prompt asking them to respond by pressing " $X$ " on the keyboard to cooperate or "M" to defect. The payoff matrix displayed the amounts of money that each player would receive given each combination of moves. To draw attention to these payoffs, we used two matrices in a pseudorandomized order within each iPDG version. One matrix resulted in the following payoffs: $\$ 10$ for defecting while one's partner, who received $\$ 0$, cooperated $(D, C) ; \$ 6$ to each player for mutual cooperation $(C, C)$; and $\$ 2$ to each player for mutual defection $(D, D)$. In the other matrix, all values were halved: $\mathrm{D}, \mathrm{C}=\$ 5, \$ 0 ; \mathrm{C}, \mathrm{C}=\$ 3$ to each player; $\mathrm{D}, \mathrm{D}=\$ 1$ to each player. In between the instructions and the first trial of the first game only, participants were asked to predict how often the average person would choose to cooperate with them in this task (between $0 \%$ and $100 \%$ of the time). Participants were not told how many rounds they would play with each partner.

\section{$\underline{2.3 \text { Statistical Analyses }}$}

The data were analyzed using SPSS version 22.0 (IBM Corp., Armonk, NY). Defection on a single trial was coded as 0 , and cooperation was coded as 1 . Average cooperation rates could range from 0 (defection on every trial) to 1 (cooperation on every trial). Average cooperation rates were calculated for participants' responses to each of the three partners separately and were normally distributed as confirmed by Shapiro-Wilk tests.

Binary logistic generalized estimating equations (GEE) models were used to analyze the data. The implementation of a GEE model allows adjusting for correlations due to repeated (binary) observations within each participant over the 12 trials played with each partner. In order 
to examine the influence of depression on cooperation over trials with the various partners, the following variables were added as predictors, depending on the analysis: BDI Score (entered as a continuous variable); the individual trials played with a given partner (Trial Order, entered as an ordinal variable); whether participants interacted with a cooperating, defecting, or unbiased partner (Partner Type, entered as a nominal variable); each participant's average cooperation across the First Three Trials with a given partner (entered as a continuous covariate); whether the iPDG involved a low or high stakes payoff matrix (Payoff, entered as a nominal variable); administration order of the three separate iPDGs (Administration Order, entered as a nominal variable); and interactions between these variables. The participant's decision (to cooperate or defect) was entered as the binary dependent variable. The variable Subject was entered as a repeated effects variable.

Using a bivariate correlation, we further tested whether participants' expectations about their partners' cooperation was related to self-reported depression levels.

\section{Results}

\subsection{Participant characteristics}

Table 1 depicts participant demographics and characteristics, including BDI and BAI scores. BDI values reported indicate that depressive symptoms ranged from the normal level to severe depression. One participant recruited as depressed appeared to be in remission based on a low BDI score (BDI value of 9), and three participants recruited as non-depressed showed BDI scores that were higher than in the normal range of mood fluctuations (BDI values of 11,18 , and 29), according to accepted BDI cut-off scores (Beck et al., 1988). Eleven participants reported current psychotropic medication use (four participants using more than one). As expected, the correlation between BDI and BAI scores was significant, $r(27)=0.69, p<0.0001$, suggesting that participants reporting higher depression levels also reported higher anxiety. 
$<<$ Insert Table 1 about here $>>$

\subsection{Depression, expectations, and cooperation over time for different partners}

We first assessed the relationship between BDI scores and participants' expectations about partner cooperation before the onset of the three games. This correlation was not significant, $r(27)=0.16, p=0.41$.

To assess cooperative decisions by participants in the task, an initial GEE model analysis was conducted using the predictors Administration Order, Partner Type, Administration Order*Partner Type interaction, and Payoff. This demonstrated a significant main effect of Administration Order, Wald $X^{2}(2)=14.22, p=0.001$; a significant main effect of Partner Type, Wald $X^{2}(2)=13.42, p=0.001$; no significant Administration Order*Partner Type interaction, Wald $X^{2}(4)=3.28, p=0.51$; and a nonsignificant, albeit trend level, main effect of Payoff (high vs. low) across iPDGs, Wald $X^{2}(1)=2.96, p=0.09$. Subsequent analyses thus combined trials from the high and low payoff matrices. Given the lack of effect of administration order on cooperating with different partners, this variable was not included in further analyses.

We next tested the influence of depression on cooperation over time with the three different partners. A GEE model with the predictors Trial Order, Partner Type, BDI Score, and their interactions demonstrated a significant main effect of Partner Type, Wald $X^{2}(2)=13.57$, $p=0.001$. Average cooperation with each partner was as follows: defecting partner $(M=0.41$, $S D=0.42)$, unbiased partner $(M=0.51, S D=0.50)$, and cooperating partner $(M=0.58, S D=0.49)$. We also observed a significant main effect of Trial Order, Wald $X^{2}(11)=56.28, p<0.0001$, and a trend toward significance for the main effect of BDI Score, Wald $x^{2}(1)=3.10, p=0.079$. The Partner Type*Trial Order, BDI Score ${ }^{\star}$ Trial Order, and Partner Type*Trial OrderBDI Score interactions were also significant, Wald $X^{2}(22)=62.51, p<0.0001$, Wald $X^{2}(11)=44.19, p<0.0001$, and Wald $X^{2}(22)=345.78, p<0.0001$, respectively. The BDI Score*Partner Type interaction was not significant, Wald $X^{2}(2)=1.96, p=0.38$. These results indicate not only that cooperation 
declined over trials, but also that cooperation varied for different partners (see Figure 1) as a function of depression severity for at least some of the partners--warranting further analyses.

It should be noted that the three partners did not differ in their pre-determined decisions until trial 3. Specifically, each partner cooperated on the first trial, and both the defecting and unbiased partners defected on the second trial. Therefore, participants could be expected to update their decision to cooperate or defect based on how each of the three partners 'behaved' only in trials 4 and after. For that reason, we analyzed participants' decisions on trials 4 through 12, after confirming that BDI scores did not affect participants' decisions with different partners in trials 1 through 3 (which could have indicated a failure in random assignment).

Results of a GEE analysis on trials 1 through 3 only with BDI Score, Partner Type, and their interaction as predictors revealed no significant main effect of Partner Type, Wald $X^{2}(2)=2.32, p=0.31$. Average cooperation rates on trials 1 through 3 played with each partner were as follows: defecting partner $(M=0.66, S D=0.48)$, cooperating partner $(M=0.63, S D=0.49)$, and unbiased partner $(M=0.69, S D=0.47)$. The main effect of BDI Score on participant cooperation in the first three trials across the three games was also not significant, Wald $X^{2}(1)=1.50, p=0.22$, and neither was the BDI Score*Partner Type interaction, Wald $X^{2}(2)=3.07$, $p=0.22$. These data suggest no influence of depression severity or partner type on participants' decisions during trials 1 through 3 , when the response tendencies of the partners could not have yet been detected.

A GEE analysis on the remaining trials, 4 through 12, with the predictors Trial Order and Partner Type as factors and BDI Score and the average cooperation rate across the First Three Trials of a given game as covariates resulted in non-significant main effects of Trial Order and Partner Type, Wald $X^{2}(8)=13.75, p=0.09$ and Wald $X^{2}(2)=1.32, p=0.52$, respectively. Average cooperation on trials 4 through 12 with each partner were as follows: defecting partner $(M=0.32$, $S D=0.47)$, cooperating partner $(M=0.56, S D=0.50)$, and unbiased partner $(M=0.44, S D=0.50)$. The model revealed significant main effects of BDI Score, Wald $X^{2}(1)=8.84, p=0.003$, and 
average cooperation rate across the First Three Trials, Wald $X^{2}(1)=15.05, p<0.001$. Moreover, we observed significant two-way interactions: Trial Order*Partner Type, Wald $X^{2}(16)=102.11$, $p<0.001$, Partner Type*BDI Score, Wald $x^{2}(2)=6.96, p=0.03$, and BDI Score*average cooperation across the First Three Trials, Wald $X^{2}(1)=4.53, p=0.03$. The Trial Order*BDI Score, Trial Order*average cooperation across the First Three Trials, and Partner Type*average cooperation across the First Three Trials interactions were non-significant $(p=0.19, p=0.49$, and $p=0.11$, respectively). All other three-way interactions (all $p<0.05$ ) and the four-way interaction BDI Score*Partner Type*average cooperation across the First Three Trials*Trial Order, Wald $X^{2}(16)=79.52, p<0.001$, were significant. These results suggest that participants' decisions to cooperate or defect varied across trials 4 through 12, for at least some of the games, as a function of the degree to which they cooperated in the first three trials of that game, their selfreported depression severity, and the response pattern of the partner encountered.

In an effort to further parse these findings, we ran separate analyses using trials 4 through 12 played with each partner type. When selecting the defecting partner only, GEE analysis resulted in a trend level main effect for Trial Order, Wald $X^{2}(8)=14.73, p=0.065$, but no significant main effects of BDI Score, Wald $X^{2}(1)=1.74, p=0.19$, or average cooperation on the First Three Trials, Wald $X^{2}(1)=0.29, p=0.59$. Trial Order*average cooperation on the First Three Trials was the only significant interaction, Wald $X^{2}(8)=23.49, p=0.003$. When selecting the cooperating partner only, GEE analysis revealed non-significant main effects of Trial Order and BDI Score, Wald $X^{2}(8)=8.35, p=0.40$ and Wald $X^{2}(1)=0.004, p=0.95$, respectively, but a significant main effect of average cooperation on the First Three Trials, Wald $X^{2}(1)=7.02$, $p=0.008$. Only the interactions Trial Order*BDI Score and Trial Order*average cooperation on the First Three Trials were also significant, Wald $X^{2}(8)=22.62, p=0.004$ and Wald $X^{2}(8)=18.78$, $p=0.02$, respectively. Finally, in a GEE analysis selecting the unbiased partner only, we observed a trending but non-significant main effect of Trial Order, Wald $X^{2}(8)=14.19, p=0.07$, and significant main effects of BDI Score, Wald $X^{2}(1)=14.08, p<0.001$, and average cooperation 
on the First Three Trials, Wald $X^{2}(1)=6.73, p=0.01$. The interactions Trial Order ${ }^{\star} B D I$ Score, BDI Score ${ }^{\star}$ average cooperation on the First Three Trials, and Trial Order*BDI Score*average cooperation on the First Three Trials were all significant, Wald $X^{2}(8)=38.76, p<0.001$, Wald $X^{2}(1)=11.78, p=0.001$, and Wald $X^{2}(8)=33.19, p<0.001$, respectively.

Taken together, these results suggest that participants' decisions to cooperate or defect across trials 4 through 12 were to some degree influenced by their decisions on the first three trials played with each partner. Cooperation rates across trials 4 through 12 played with the cooperating and unbiased partners varied for participants differing in depression levels, but this was not the case when playing with the defecting partner. As seen in Figure 2, more severe depression was associated with steadier cooperation over trials played with the cooperating partner (panel 2B). Results for the unbiased partner (panel 2C) appear to suggest that participants with more severe depression made more volatile decisions throughout trials 4 through 12, which was further influenced by their decisions on the first three trials.

Given that the majority of our sample (72\%) consisted of females and that the prevalence of depression is typically higher among women than men (Kessler, 2003), results of these analyses including only the 21 females in our sample can be found in the Supplementary Materials.

$<<$ Insert Figures 1 and 2 about here $>>$

\subsection{Potential role of anxiety}

Given the high correlation between depression and anxiety and our results suggesting some influence of BDI scores on average cooperation across the three iPDGs (i.e. borderline significant and significant main effects of BDI Score in the analyses including all 12 trials and trials 4 through 12, respectively), we repeated the GEE models on (1) all 12 trials and (2) trials 4 through 12, across all three games, using anxiety as a predictor (i.e. BAI Score rather than BDI 
Score entered as a continuous variable). Results of the GEE analysis including all 12 trials revealed significant main effects of Trial Order and Partner Type, Wald $X^{2}(11)=38.19, p<0.001$ and Wald $X^{2}(2)=15.73, p<0.001$, respectively. We did not observe a significant main effect of BAI Score, Wald $x^{2}(2)=0.30, p=0.59$. Furthermore, the interaction Partner Type*BAI Score was not significant, Wald $X^{2}(2)=3.15, p=0.21$. The interactions Trial Order ${ }^{\star}$ Partner Type, Trial Order*BAI Score, and Trial Order*Partner Type ${ }^{\star} B A I$ Score were significant, Wald $X^{2}(22)=57.23$, $p<0.001$, Wald $X^{2}(11)=20.58, p<0.001$, and Wald $X^{2}(22)=402.65, p<0.001$, respectively.

Next, we ran a GEE analysis on trials 4 through 12 only, adding average cooperation across the First Three Trials as a covariate. This resulted in non-significant main effects of Trial Order, Partner Type, and BAI Score, but a significant main effect of average cooperation across the First Three Trials, Wald $X^{2}(8)=6.58, p=0.58$, Wald $X^{2}(2)=2.79, p=0.25$, Wald $X^{2}(1)=0.41$, $p=0.52$, and Wald $X^{2}(1)=7.59, p=0.006$, respectively. The significant interactions involving BAI Score were (other interactions are not reported due to overlap with BDI analyses): BAI Score*Partner Type, BAI Score ${ }^{\star}$ Trial OrderPartner Type, BAI Score*Partner Type*average cooperation across First Three Trials, BAI Score ${ }^{\star}$ Trial Order ${ }^{\star}$ Partner Type*average cooperation across First Three Trials, Wald $X^{2}(2)=10.13, p=0.006$, Wald $X^{2}(16)=74.21, p<0.001$, Wald $X^{2}(2)=9.75, p=0.008$, Wald $X^{2}(16)=58.04, p<0.001$, respectively. The interactions BAI Score ${ }^{\star}$ Trial Order, BAI Score*average cooperation across First Three Trials, BAI Score*Trial Order*average cooperation across First Three Trials were non-significant, Wald $X^{2}(8)=7.92, p=0.44$, Wald $X^{2}(1)=0.09, p=0.77$, Wald $X^{2}(8)=10.82, p=0.21$, respectively.

In an effort to limit the number of analyses on the same dataset and given the absence of BAI main effects, we did not further analyze the effect of BAI Score on cooperation with each of the three partner types separately. 


\section{Discussion}

We first showed that participants, regardless of depression symptoms, distinguished behaviorally between different partners encountered in the iPDGs. Across all 12 trials, participants cooperated significantly less with the defecting partner than with the others, dropping to an average of $41 \%$ cooperation with the defecting partner from $51 \%$ and $58 \%$ with the unbiased and cooperating partners, respectively. Average cooperation declined over repeated interactions, consistent with previous research (Andreoni and Miller, 1993; Silverstein et al., 1998). Figure 1 shows that this decline in cooperation occurred most prominently when playing with the defecting partner and the least prominently when playing with the cooperating partner. These data indicate that participants accurately adjusted their behavior to the partners they encountered.

The main focus of our study, however, was to evaluate whether self-reported depression severity was associated with a steeper decline in cooperation (toward defection) across trials when interacting with a partner exhibiting a defecting play strategy. This appeared not to be the case, as analyses revealed no significant effect of depression severity on cooperative decisions made when interacting with the defecting partner in trials 4 through 12 , when this partner demonstrated a tendency to defect. This finding supports the idea that these participants were able to adequately adjust their interpersonal decision making strategy based on the social feedback provided by the defecting partner. This is in line with work by Murphy et al. (2003), demonstrating that although inaccurate negative feedback disrupted performance in depression, accurate negative feedback did not.

A more unexpected finding was the association between more severe levels of depression and maintained levels of cooperation across trials 4 through 12 played with the cooperating partner. We recognized that while some participants might have aimed to establish mutual cooperation in this condition, others might have realized that defecting against a predominantly cooperative partner could result in higher personal monetary gains on a given 
trial. It is noteworthy to highlight, as displayed in Figure 2B and supported by the significant interaction between trial order and depression severity, the reduction in cooperative decisions halfway through the game in individuals reporting no depression (a BDI score of 0 or 1 ), while individuals reporting higher depression levels appear more inclined toward mutual cooperation across repeated interactions. This suggests that depression severity may influence interpersonal play strategy and that individuals reporting no mood fluctuations take greater advantage of a cooperating partner. This finding appears to be somewhat in line with McClure et al. (2007), who reported more cooperative behavior with cooperative partners, indicative of a prosocial behavioral style, in adolescents with anxiety and depression compared to adolescents without these disorders.

Similarly surprising was our finding that depression severity significantly influenced cooperation over repeated interactions with the unbiased partner. As seen in Figure $2 \mathrm{C}$, it appears that individuals reporting more severe depressive symptoms displayed more volatile decisions across trials 4 through 12, alternating between cooperation and defection, when interacting with this partner. In contrast, individuals with no or lower depression levels appeared to more gradually reduce cooperation after the first three trials, when this partner's unique decision pattern could be discerned. This could reflect that individuals with more severe depression relied more heavily on the trial-by-trial social feedback provided, e.g. more tit-for-tat decisions or trial-and-error behavior to get to 'know' this partner. This is inconsistent with a study by Cella et al. (2010), who found that depression was associated with reduced updating of decisions based on reward and punishment feedback in the lowa Gambling Task, a non-social risky decision-making paradigm. The discrepancy may be explained by the fundamental differences between the lowa Gambling Task and our task, the latter being social in nature and offering no clearly advantageous decision options. Conversely, our observation also demonstrates that individuals with no or less severe depression tended to opt for the less risky option, thus defecting. 
Our results further show that initial decisions on trials 1 through 3 , before partner play tendencies became apparent, did not differ for the three different partners, nor were these initial decisions influenced by depression severity. This confirms that the three iPDGs were treated as separate interactions, although our analysis of administration order does show that having previously played an iPDG had some influence on subsequent iPDG behavior. Responding on these initial trials may reflect other motives such as a willingness to take a risk, trust others or act prosocially, a desire to prevent potential conflict, as well as participants' expectations about social interactions when starting to get to know each partner.

A common finding within each of the three separate games was that participants' decisions made on the initial trials played with a given partner influenced their cooperation over the course of subsequent trials of that game. Thus, across depression severity levels, participants' decisions in response to cooperation or defection by a given partner were influenced by their own initial tendencies to cooperate. This might not be surprising since, in all three games, participant cooperation during trials 1 through 3 was higher than during the remaining trials 4 through 12 . This further supports our finding, consistent with prior research, of an overall decline in cooperation over repeated interactions within each game (Andreoni and Miller, 1993; Silverstein et al., 1998). Our observation of a significant interaction between depression severity, initial cooperation in trials 1 through 3 , and subsequent cooperation in trials 4 through 12 with the unbiased partner suggests that participants varying on depression severity differed in their decisions during the early trials, which subsequently affected their responses on the remaining trials, 4 through 12 (see Figure $2 \mathrm{C}$ ).

Taken together, our findings demonstrate that when playing with the defecting partner (who presented negative directional feedback for participants to use in adjusting their decisions), participants' response patterns were influenced by their initial choices in reaction to feedback from individual trials, but not by differences in depression levels. However, when playing with the cooperating partner (who provided positive directional feedback), participants' 
choices on the first three trials and their depression severity both influenced cooperation over the course of subsequent trials, suggesting a prosocial behavioral style in those with higher levels of depression. When interacting with the unbiased partner (with no clear directional feedback present), participants' responses during trials 4 through 12 were influenced by their initial decisions, depression severity, and subsequent feedback provided by this partner, indicative of more volatile decisions in response to the unpredictable behavior of this partner. Nonetheless, participants were able to adapt their behavior according to the social information encountered in these interactions over time once their partners' predominant response patterns could be discerned. Our data do not seem to indicate a general unwillingness to take risks (i.e. cooperation), preconceived negative expectations, or wanting to avoid conflict.

Nonetheless, our data contrasts prior research on the PDG demonstrating less cooperative behavior or intentions (Clark et al., 2013; Pulcu et al., 2015; Surbey, 2011), as well as more aggressive behavior after being betrayed (Haley and Strickland, 1986), in individuals reporting greater depression. It is possible that differences in the characteristics of our sample compared to previous studies contributed to this discrepancy. Perhaps the most prominent difference between the present study and previous work, however, is that we manipulated the repeated interpersonal play tendencies that participants encountered in their partners. In contrast, previous work on the iPDG has often employed a tit-for-tat-like strategy (i.e. Clark et al., 2013; Pulcu et al., 2015) in which partners' decisions mirror those of the participants, rather than presenting pre-structured play patterns to which participants respond.

Our data on participants' expectations did not show a relationship with reported depression levels, nor do our results seem to support prior studies showing that individuals with depression may more quickly learn to avoid risky decisions (Smoski et al., 2008). This previously-reported risk aversion might be attributed to anxiety that is often comorbid with depression (Eisenberg et al., 1998; Maner et al., 2007). However, unlike our results on depression severity, our analyses on the influence of anxiety on cooperation across all trials, as 
well as across trials 4 through 12 , did not reveal significant main effects of anxiety severity. We did observe some significant interaction effects involving anxiety severity, including a significant effect of anxiety severity on cooperation across trials depending on the particular partner encountered, which were also present in our analyses on depression. The similarity of interaction effects for anxiety and depression is not very surprising given the high correlation between these scores. Our results on anxiety should therefore be interpreted with caution. Interestingly, social anxiety has been associated with difficulties in maintaining cooperative relationships in the iPDG. Compared to participants with lower or no social anxiety, those with higher social anxiety have shown greater cooperation (Rodebaugh et al., 2011) and reduced behavioral change (Rodebaugh et al., 2013) in response to partner defection. Furthermore, neuroimaging research suggests that individuals with social anxiety disorder do not display the expected activation patterns in the medial prefrontal cortex thought to underlie accurate interpretation of a partner's intentions (Sripada et al., 2009), nor the expected striatal activity thought to reflect establishing cooperative relationships (Sripada et al., 2013), seen in healthy controls. Although difficult to parse, further research is needed to shed more light on the unique contributions of depression and anxiety to interpersonal decision making patterns.

Several limitations of the present study should be noted. We chose to assess response differences as a function of participants' current, self-reported depressive symptoms, whether or not they had received a (lifetime) clinical diagnosis. As a result, several participants met criteria for one or more psychological concerns based on the MINI assessment, and others appeared to have a depressive disorder in (partial) remission. Second, despite pseudorandomization, the sequential administration of three separate iPDGs resulted in less participant cooperation in later games. Nonetheless, this did not significantly influence the differential effects of the three different partners on cooperation rates. Third, we included 12 trials in each iPDG version in which decisions were dichotomous (cooperate or defect), based on a prior study using a somewhat similar design (Chang et al., 2010). Our findings might have been different had we 
examined real social interactions without the use of deception regarding the computerized nature of the partner or if we had used a more dimensional approach to participants' willingness to cooperate (e.g. cooperate not at all, $0 \% ; 25 \% ; 50 \% ; 75 \%$; or fully, $100 \%$ ). More specifically, such a dimensional willingness to cooperate would be a more sensitive measure of the participants' doubt about the likelihood that a partner would cooperate or defect. Similarly, our task might have revealed additional information about whether individuals who vary in their selfreported depression symptom severity (persistently) apply different response strategies (e.g. stick to defecting after a certain number of partner defections) had we included more trials.

Finally, the current sample size of 29 participants is small. This prohibited analysis of the effects of comorbidity or the use of medication, which are important confounds. Furthermore, although we chose a statistical approach that we feel best captures the nature of our data (i.e. analyzing responses over trials while taking into account adjusting for correlations due to repeated (binary) observations within each participant), it may appear overly complex given the small sample size, and conducting multiple analyses on the same data typically inflates type I error (see Supplementary Materials for an assessment of power within this study). Future studies should examine larger samples, including individuals with depression confirmed by a clinician's diagnosis, and assess the effects of psychoactive medication. More research on the influence of anxiety, or other comorbidities, is also warranted given the high correlation between severity of anxiety and depression both in this sample and in existing research (e.g. Julian, 2011). Consequently, our results involving anxiety should be interpreted with caution.

In sum, our iPDG design aimed to assess context-appropriate decision making in a dynamic interpersonal setting. Our data shows that participants altered their initial decisions once their partners' predominant response patterns could be discerned, suggesting that participants were able to adapt their behavior according to the social information encountered in these interactions over time. Additionally, severity of self-reported depression symptoms was associated with different patterns of responding over repeated interactions when interacting with 
a cooperating or unbiased partner: more severe depression was associated with more consistent cooperation over repeated interactions with the cooperating partner and seemingly more volatile decision making in response to repeated, but inconsistent, feedback from the unbiased partner. These findings provide insight into consequential decision making in individuals experiencing symptoms of depression in a social context and further demonstrate the effectiveness of tasks borrowed from game theory to assess complex social behavior.

\section{Acknowledgements}

This material is based in part upon work supported by the National Science Foundation under Grant No. NSF 1156090; PIs: M. Dean and P. Ortoleva. We would like to thank R. Jones for his work on a power assessment of the obtained data. 


\section{References}

American Psychiatric Association, 2013. Diagnostic and Statistical Manual of Mental Disorders, fifth ed. Washington, DC.

Andreoni, J., Miller, J. H., 1993. Rational cooperation in the finitely repeated prisoner's dilemma: Experimental evidence. The Economic Journal 103, 570-585.

Argyris, C., 1965. Explorations in interpersonal competence: I. Journal of Applied Behavioral Science 1, 58-83.

Axelrod, R., Hamilton, W. D., 1981. The evolution of cooperation. Science 211, 1390-1396.

Beck, A. T., Rush, A. J., Shaw, B. E, Emery, G., 1979. Cognitive Therapy of Depression. New York, New York.

Beck, A. T., Steer, R. A., 1990. Manual for the Beck Anxiety Inventory. San Antonio, Texas.

Beck A. T., Steer, R. A., Garbin, M. G. J., 1988. Psychometric properties of the Beck Depression Inventory Twenty-five years of evaluation. Clinical Psychology Review 8, 77100.

Berg, J., Dickhaut, J., McCabe, K., 1995. Trust, Reciprocity, and Social History. Games and Economic Behavior 10, 122-142.

Brittlebank, A. D., Scott, J., Williams, J. M., Ferrier, I. N., 1993. Autobiographical memory in depression: State or trait marker? The British Journal of Psychiatry 162, 118-121.

Canivez, G. L., Konold, T. R., Collins, J. M., Wilson, G., 2009. Construct validity of the Wechsler Abbreviated Scale of Intelligence and Wide Range Intelligence Test: Convergent and structural validity. School Psychology Quarterly 24, 252-265.

Camerer, C., 2003. Behavioral game theory: Experiments in strategic interaction. Princeton University Press, Princeton, New Jersey.

Cella, M., Dymond, S., Cooper, A., 2010. Impaired flexible decision-making in major depressive disorder. Journal of Affective Disorders 124, 207-210. 
Chang, L. J., Doll, B. B., van 't Wout, M., Frank, M. J., Sanfey, A. G., 2010. Seeing is believing: Trustworthiness as a dynamic belief. Cognitive Psychology 61, 87-105.

Clark, L., Chamberlain, S. R., Sahakian, B. J., 2009. Neurocognitive mechanisms in depression: Implications for treatment. Annual Review of Neuroscience 32, 57-74.

Clark, C. B., Thorne, C. B., Hardy, S., Cropsey, K. L., 2013. Cooperation and depressive symptoms. Journal of Affective Disorders 150, 1184-1187.

Delgado, M. R., Frank, R. H., Phelps, E. A., 2005. Perceptions of moral character modulate the neural systems of reward during the trust game. Nature Neuroscience 8, 1611-1618.

Eisenberg, A. E., Baron, J., Seligman, M. E., 1998. Individual differences in risk aversion and anxiety. Psychological Bulletin 87, 245-251.

Elliott, R., Sahakian, B. J., Herrod, J. J., Robbins, T. W., Paykel, E. S., 1997. Abnormal response to negative feedback in unipolar depression: Evidence for a diagnosis specific impairment. Journal of Neurology, Neurosurgery \& Psychiatry 63, 74-82.

Erickson, K., Drevets, W.C., Clark, L., Cannon, D.M., Bain, E.E., Zarate, C.A., Charney, D.S., Sahakian, B.J., 2005. Mood-congruent bias in affective go/nogo performance of unmedicated patients with major depressive disorder. American Journal of Psychiatry $162,2171-2173$

Haley, W. E., Strickland, B. R., 1986. Interpersonal betrayal and cooperation: Effects on selfevaluation in depression. Journal of Personality and Social Psychology 50, 386-391.

Harlé, K. M., Allen, J. J., Sanfey, A. G., 2010. The impact of depression on social economic decision making. Journal of Abnormal Psychology 119, 440-446.

IBM Corp. Released 2013. IBM SPSS Statistics for Windows, Version 22.0. Armonk, NY: IBM Corp.

Joiner, T. E., 1995. The price of soliciting and receiving negative feedback: Self-verification theory as a vulnerability to depression theory. Journal of Abnormal Psychology 104, 364372. 
Joormann, J., Teachman, B.A., Gotlib, I.H., 2009. Sadder and less accurate: Memory for negative material in depression. Journal of Abnormal Psychology 118, 412-417.

Judd, L. L., Paulus, M. P., Wells, K. B., Rapaport, M. H., 1996. Socioeconomic burden of subsyndromal depressive symptoms and major depression in a sample of the general population. American Journal of Psychiatry 153, 1411-1417.

Julian, L. J. 2011. Measures of anxiety: State-Trait Anxiety Inventory (STAI), Beck Anxiety Inventory (BAI), and Hospital Anxiety and Depression Scale-Anxiety (HADS-A). Arthritis Care \& Research 63, S467-S472.

Kessler, R.C., 2003. Epidemiology of women and depression. Journal of affective disorders 74, $5-13$.

Kessler, R.C., Berglund, P.A., Demler, O., Jin, R., Walters, E.E., 2005. Lifetime prevalence and age-of-onset distributions of DSM-IV disorders in the National Comorbidity Survey Replication (NCS-R). Archives of General Psychiatry 62, 593-602.

Libet, J. M., Lewinsohn, P. M., 1973. Concept of social skill with special reference to the behavior of depressed persons. Journal of Consulting and Clinical Psychology 40, 304312.

Maner, J. K., Richey, J. A., Cromer, K., Mallott, M., Lejuez, C. W., Joiner, T. E., Schmidt, N. B., 2007. Dispositional anxiety and risk-avoidant decision-making. Personality and Individual Differences 42, 665-675.

McClure, E. B., Parrish, J. M., Nelson, E. E., Easter, J., Thorne, J. F., Rilling, J. K., Ernst, M., Pine, D. S., 2007. Responses to conflict and cooperation in adolescents with anxiety and mood disorders. Journal of Abnormal Child Psychology 35, 567-577.

Murphy, F.C., Michael, A., Robbins, T.W., Sahakian, B.J., 2003. Neuropsychological impairment in patients with major depressive disorder: The effects of feedback on task performance. Psychological Medicine 33, 455-467. 
Murphy, F.C., Sahakian, B.J., Rubinsztein, J.S., Michael, A., Rogers, R.D., Robbins, T.W., Paykel, E.S., 1999. Emotional bias and inhibitory control processes in mania and depression. Psychological Medicine 29, 1307-1321.

Nelson, R. E., Craighead, W. E., 1977. Selective recall of positive and negative feedback, selfcontrol behaviors, and depression. Journal of Abnormal Psychology 86, 379-388.

Nepon, T., Flett, G. L., Hewitt, P. L., Molnar, D. S., 2011. Perfectionism, negative social feedback, and interpersonal rumination in depression and social anxiety. Canadian Journal of Behavioural Science 43, 297-308.

Nowak, M. A., 2006. Five rules for the evolution of cooperation. Science 314, 1560-1563.

Nowak, M., Sigmund, K., 1993. A strategy of win-stay, lose-shift that outperforms tit-for-tat in the Prisoner's Dilemma game. Nature 364, 56-58.

Ortmann, A., Tichy, L. K., 1999. Gender differences in the laboratory: Evidence from prisoner's dilemma games. Journal of Economic Behavior \& Organization 39, 327-339.

Psychological Corporation, 1999. Wechsler Abbreviated Scale of Intelligence. San Antonio, Texas.

Psychology Software Tools, Inc. [E-Prime 2.0]. 2012.

Pulcu, E., Thomas, E. J., Trotter, P. D., McFarquhar, M., Juhasz, G., Sahakian, B. J., Deakin, I.M., Zahn, R., Elliott, R., 2015. Social-economical decision making in current and remitted major depression. Psychological Medicine 45, 1301-1313.

Rodebaugh, T. L., Klein, S. R., Yarkoni, T., Langer, J. K., 2011. Measuring social anxiety related interpersonal constraint with the flexible iterated prisoner's dilemma. Journal of Anxiety Disorders 25, 427-436.

Rodebaugh, T. L., Shumaker, E. A., Levinson, C. A., Fernandez, K. C., Langer, J. K., Lim, M. H., Yarkoni, T., 2013. Interpersonal constraint conferred by generalized social anxiety disorder is evident on a behavioral economics task. Journal of Abnormal Psychology 122, 39-44. 
Rubinow, D.R., Post, R.M., 1992. Impaired recognition of affect in facial expression in depressed patients. Biological Psychiatry 31, 947-953.

Sally, D., 1995. Conversation and cooperation in social dilemmas a meta-analysis of experiments from 1958 to 1992 . Rationality and society 7, 58-92.

Sheehan, D.V., Lecrubier, Y., Harnett-Sheehan, K., Amorim, P., Janavs, J., Weiller, E., Hergueta, T., Baker, R., Dunbar, G.,1998. The Mini International Neuropsychiatric Interview (M.I.N.I.): The development and validation of a structured diagnostic psychiatric interview for the DSM-V and ICD-10. Journal of Clinical Psychiatry 59, 22-33.

Silverstein, A., Cross, D., Brown, J., Rachlin, H., 1998. Prior experience and patterning in a prisoner's dilemma game. Journal of Behavioral Decision Making 11, 123-138.

Smoski, M. J., Lynch, T. R., Rosenthal, M. Z., Cheavens, J. S., Chapman, A. L., Krishnan, R. R., 2008. Decision-making and risk aversion among depressive adults. Journal of Behavior Therapy and Experimental Psychiatry 39, 567-576.

Sripada, C. S., Angstadt, M., Banks, S., Nathan, P. J., Liberzon, I., Phan, K. L., 2009. Functional neuroimaging of mentalizing during the trust game in social anxiety disorder. NeuroReport 20, 984-989.

Sripada, C., Angstadt, M., Liberzon, I., McCabe, K., Phan, K. L., 2013. Aberrant reward center response to partner reputation during a social exchange game in generalized social phobia. Depression and Anxiety 30, 353-361.

Surbey, M. K., 2011. Adaptive significance of low levels of self-deception and cooperation in depression. Evolution and Human Behavior 32, 29-40.

van 't Wout, M., Sanfey, A. G., 2008. Friend or foe: The effect of implicit trustworthiness judgments in social decision-making. Cognition 108, 796-803. 


\section{Tables and Figures}

Table 1. Participant demographics.

\begin{tabular}{|c|c|}
\hline & Mean (SD), or Number of Participants \\
\hline Age in years & $32.28(11.77)$ \\
\hline Female:Male Ratio & $21: 8$ \\
\hline Education in years & $13.76(1.90)$ \\
\hline Parental education in years & $13.89(2.82)$ \\
\hline Ethnicity (\# participants) & $\begin{array}{l}\text { Caucasian (12); African-American (4); Hispanic (7); Asian (1); } \\
\text { mixed-race or other (5) }\end{array}$ \\
\hline WASI 2-test IQ & $97.72(14.67)$ \\
\hline BDI Scores & $13.79(12.85)$ \\
\hline BAI Scores & $18.07(15.83)$ \\
\hline $\begin{array}{l}\text { MINI criteria (\# } \\
\text { participants) }\end{array}$ & $\begin{array}{l}\text { GAD (1); Major Depressive episode (6), as well as (hypo)manic } \\
\text { episode (3); (hypo)manic episode only (2); panic disorder with or } \\
\text { without agoraphobia or agoraphobia without panic disorder (8); } \\
\text { OCD (1); PTSD (3); alcohol abuse (5). }\end{array}$ \\
\hline Medication (\# participants) & $\begin{array}{l}\text { Beta blocker (1); Anticonvulsant (1); SSRI and non-SSRI } \\
\text { Antidepressants (9); Benzodiazepines (2); Atypical antipsychotic } \\
\text { (1); Psychostimulant (1) }\end{array}$ \\
\hline
\end{tabular}

BDI: Beck Depression Inventory (Beck et al., 1979); BAI: Beck Anxiety Inventory (Beck and Steer, 1990); WASI: Wechsler Abbreviated Scale of Intelligence (Psychological Corporation, 1999); MINI: Mini International Neuropsychiatric Interview; GAD: generalized anxiety disorder; OCD: obsessive-compulsive disorder; PTSD: posttraumatic stress disorder. 
Figure 1. Average cooperation on all 12 trials in each iterated Prisoner's Dilemma Game, separately for the cooperating, defecting, and unbiased partners. Error bars represent standard error

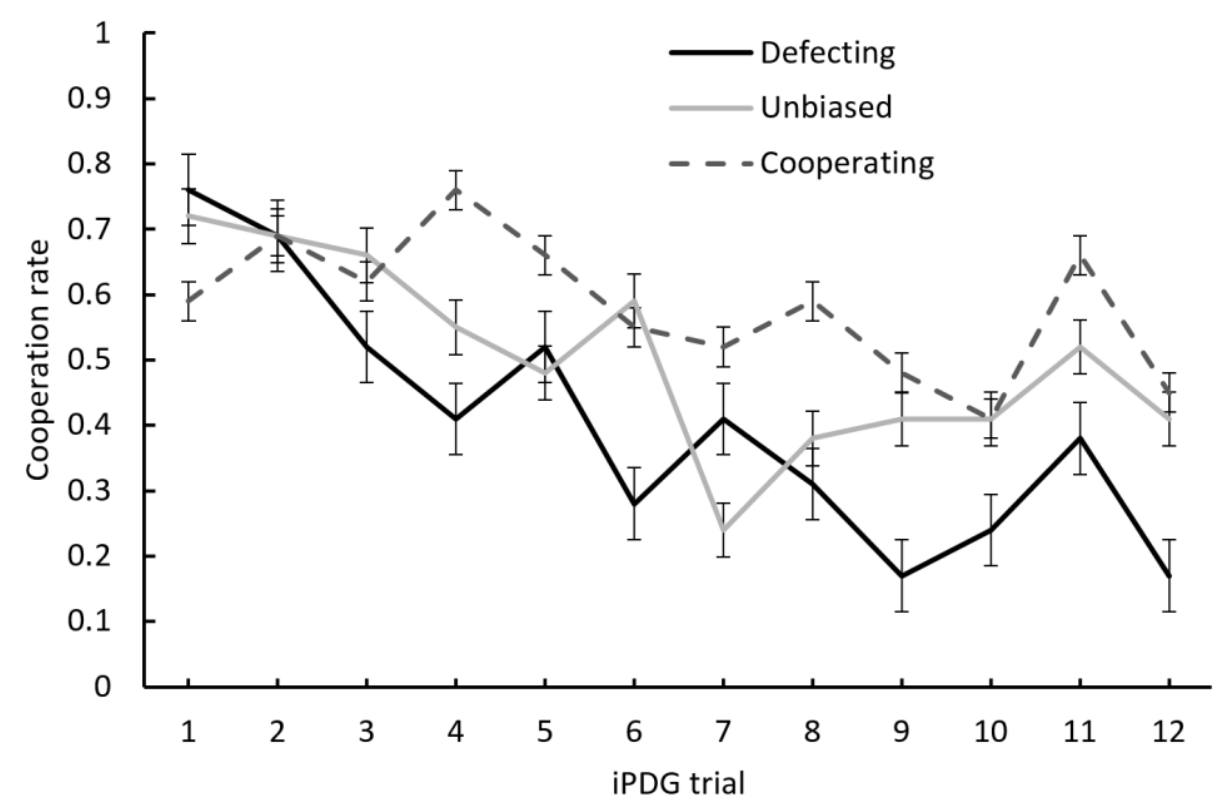


Figure 2. Average cooperation on all 12 trials in the iterated Prisoner's Dilemma Game played with the (A) defecting, (B) cooperating, and (C) unbiased partner, as a function of BDI scores depicted as quartiles (for graphing purposes only): lowest $25 \%$ of BDI scores (scores: $0-1$ ), $2^{\text {nd }}$ $25 \%$ of BDI scores (scores: $2-11$ ), $3^{\text {rd }} 25 \%$ of BDI scores (scores: $15-18$ ), and highest $25 \%$ of BDI scores (scores: 22-43). Vertical light grey line represents the distinction between the first three trials and trails 4 through 12 .
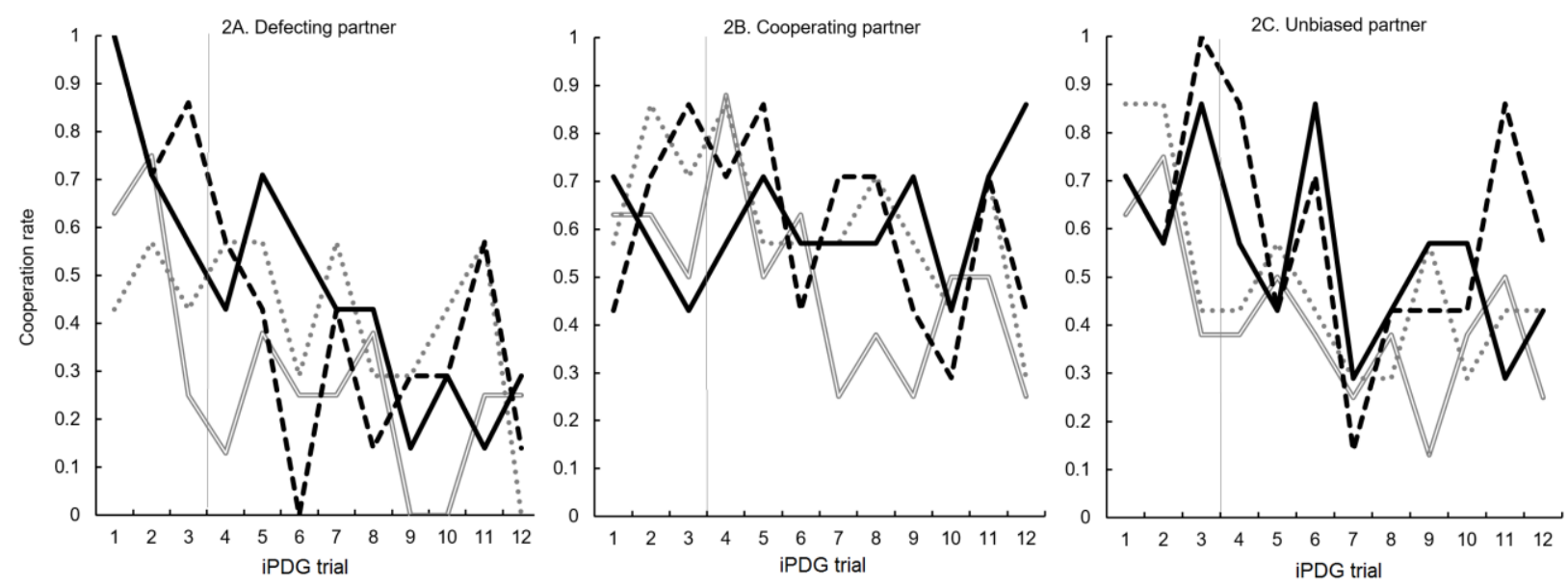

lowest $25 \%$ (score $0-1$ )

.... 2nd lowest 25\% (scores 2-11)

- - 3rd 25\% (scores 15-18)

— highest 25\% (scores 22-43) 